\title{
Protection contre l'emballement des Turbines hydrauliques
}

Pour connaitre les conditions d'installation avec une pression moyenne et avec une conduile très courte (disposition souvent cinployée ces derniers temps), nous allons étudier les centrales installées sur la rivière "Mittlere Isar "Eitting (fig. 6) et Aufkirchen. Cette dernière est semblable à tous points de vue à Eitting, sauf en ce qui concerne la longueur de la conduite d'eau. Voici leurs caracléristiques :

\section{Eilting}

Chute $\mathrm{H} \ldots \ldots \ldots$.......

Puissance $N \ldots \ldots$. . .

Vitesse $n \ldots \ldots \ldots \ldots$.

Vitesse $n_{0 t} \ldots \ldots \ldots$.

$\mathrm{GD}^{2} \mathrm{tm}^{2}, \ldots \ldots \ldots$

Itm $\sec ^{2} \ldots \ldots \ldots \ldots$

Couple $\mathrm{M}_{\mathrm{d}} \mathrm{kgm} \ldots \ldots$.

Couple de perle $\mathrm{H}_{\mathrm{v}} \mathrm{kgm}$

Diamètre de la con-

duite $\mathrm{D} m . . . . . . . .$.

Longueur de la con-

duite $\mathrm{Lm} . . . . . . . .$.

Temps d'écoulem. $t$ sec.

\section{Aufkirchen}

$25 \mathrm{~m} . \quad 25 \mathrm{~m}$.

$12.700 \mathrm{CV} .12 .700 \mathrm{CV}$.

$166,7 \quad 166,7$ normal

$344 \quad 344$ maximum

$800 \quad 800$ (turbine y inclus)

$20,4 \quad 20,4$

$54.500 \quad 54.500$ normal

$3.270 \quad 3.270$

$5 \quad 5$

$34 \quad 25$

139,53
La vitesse maximum par contre est donnée par la diminution correspondante de la chute suivant les courbes $n_{0}{ }^{1}(E)$ pour Eitting et $n_{0}{ }^{1}$ (A) pour Aufkirchen. Pour la construction de ces courbes de vitesse maximum, l'explication donnée pour la figure 5 est aussi valable ici. A cause de l'écoulement plus rapide de l'eau dans la conduite plus courte d'Aufkirchen, sa vitesse diminue plus vite, comme le moment daccélération $\mathrm{MI}_{\mathrm{b}} \mathrm{A}$ pour Aufkirchen diminuait déjà plus vite. Il en résultait que la courbe de vitesse $n_{0}{ }^{1}$ A était un peu en dessous de celle d'Eitting $n_{0}{ }^{1} \mathrm{E}$.

Les points de coupure $H_{e}$, respectivement $H_{a}$, de la courbe $\mathrm{n}_{0}$ avec la courbe $n_{0}{ }^{1}$ indiquent les valeurs maximum de l'augmentation de vitesse : 280 tours pour Eitting et 274 tours pour Aufkirchen; c'est-à-dire pratiquement sans différence. On reremarque par contre que pour les conduites en acier ou des conduites en béton d'une longueur très courte, le temps d'emballement peut devenir suffisamment court, de sorte que les points de coupure des courbes $n_{0}$ et $n_{0}{ }^{1}$ se rapprochent de la vitesse de 1,41 de la vitesse normale ou en dessous.

Le point $\mathrm{H}$ (voir figure 8 ) représentant le point limite pour une surcharge admise $n_{\mathbf{h}}$ se construit par deux moyens. H se trouve d'une part sur la courbe $n_{0}{ }^{1}$, indiquant la vitesse maximum pour

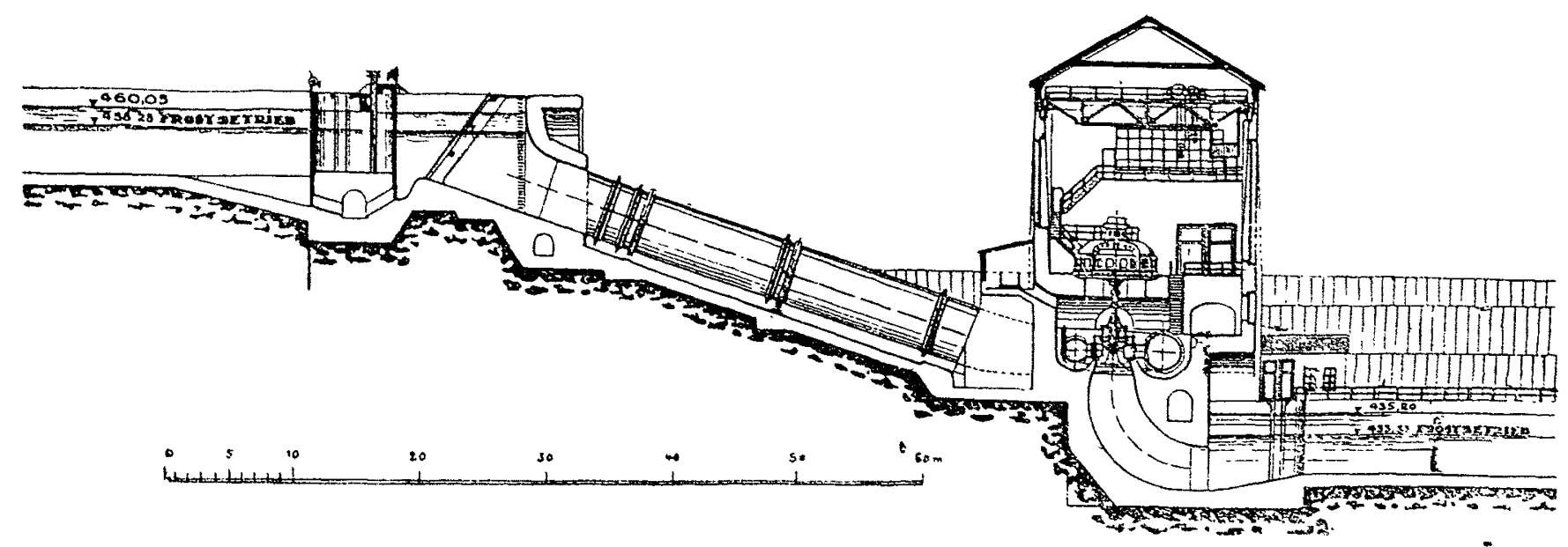

Fïg. 6. - Centralè à moyenne pression. Coupe transversale de la Centrale Eitting sur l'Isar (Barrère)

L'augmentation de la vitesse est identique pour les deux centrales, elle est indiquée par la courbe $n_{0}$ de la figure $n^{0} 7$. Les vitesses d'emballement par contre sont différentes à cause de la diminution plus ou moins rapide de la hauteur de pression, résul$\operatorname{tant}$ du volume d'eau différent des conduites. Pour la centrale Filting avec la conduite plus longue, la vitesse s'accélère quand pour le point $\mathrm{C}$ et pour une augmentation de vitesse de $20 \%$ (alteinte après $11 / 2 \mathrm{sec}$. comptée à partir du moment de l'emballement) la fermeture rapide des vannes en amont, commence. La vitesse augmente dans un intervalle de 3,4 secondes (à partir du commencement d'emballement) et atteint la valeur 1,41 de la vitesse normale comme le montre la courbe $n_{0} \mathrm{E}$, correspondant à la diminution du couple.

(Voir description de ces centrales dans Die Wasserkraft 1921). Description par Krieger. chaque instant du niveau de l'eau, c'est-à-dire à la distance $t_{\mathrm{h}}$ (mesuré en secondes) du moment de dépassement de la vitesse normale. Les caractéristiques d'une centrale nous permettent de calculer pour chaque point $H$ le temps $t_{\mathrm{b}}$ pour lequel le niveau d'eau a atteint la hauteur $\mathrm{H}_{1}=\mathrm{H}_{\mathrm{h}}$ et correspondant au rapport $\frac{n^{\text {ht }}}{n_{\mathrm{t}}}=\sqrt{\frac{\overline{\mathrm{H}_{\mathrm{h}}}}{\mathrm{H}}}$ suivant la formule (1) :

$$
\begin{array}{cc} 
& t_{\mathrm{h}}=0,452 \frac{\mathrm{F}}{f}\left(V \overline{\mathrm{H}}-V \overline{\mathrm{H}_{\mathrm{h}}}\right) . \\
\text { et }: & V \overline{\mathrm{H}_{\mathrm{h}}}=\frac{n_{\mathrm{ht}}}{n_{\mathrm{t}}} \cdot V \overline{\mathrm{H}}
\end{array}
$$

Il est à noter que la vitesse d'emballement de la turbine seule est plus élevée que celle du groupe.

Le rapport habituel de la courbe de perte $M$ à la courbe du couple est environ : 
Pour la marche à vide:

$$
n_{0}=0,94 \grave{a} 0,92 \cdot n_{0 \mathrm{t}}
$$

et pour la vilesse 1,41 de la, vitesse normale :

$$
n_{1,41}=0,96 \text { à } 0,93 \cdot n_{\mathrm{t}}
$$

et la valeur moyenne :

$$
V \overline{\mathrm{H}_{\mathrm{h}}}=\frac{0,93 \cdot n_{\mathrm{ht}}}{n_{0} \cdot 0,945} \cdot V \overline{\mathrm{H}}=0,985 \cdot \frac{n_{\mathrm{ht}}}{n_{0}} \cdot V \overline{\mathrm{II}}
$$

Il y a maintenant deux possibilités d'abaisser le point H. Premièrement par le moyen proposé par Attlmayr et Jasse en ralen-

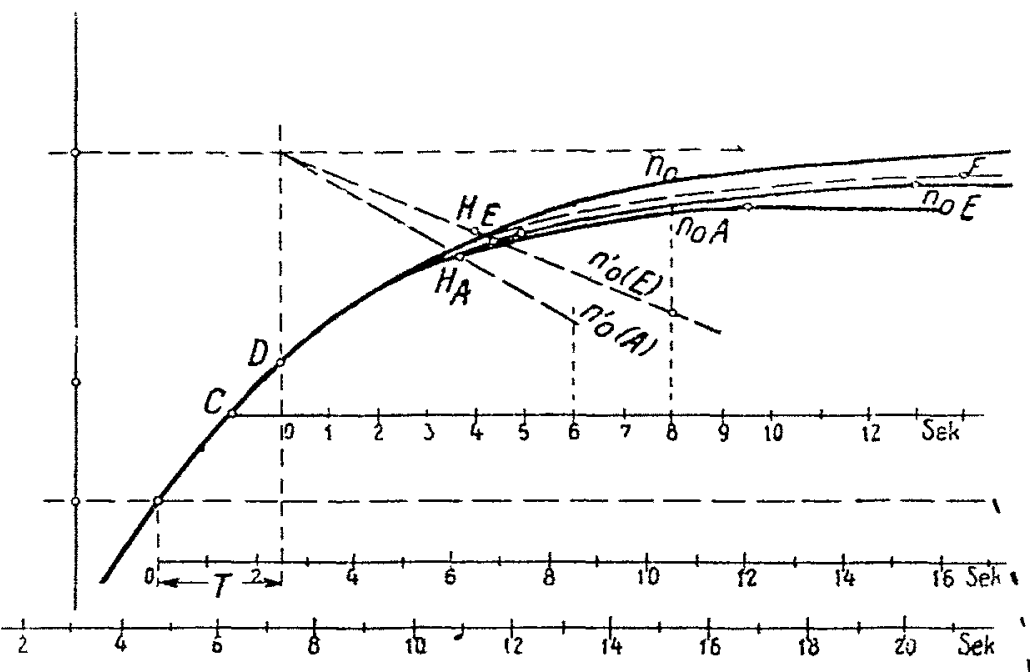

Fig. 7.- Caractéristiques de turbine à moyenne pression avec conduite en acier

tissant l'augmentation de la vitesse par l'emploi d'un moment d'inertie plus grand, exigeant un temps d'emballement proportionnellement plus long et $t_{\mathrm{h}}$ plus grand. Une augmentation relativement petite du moment d'inertie fait descendre sensiblement le point de coupure $\mathrm{H}_{1}$ de la courbe $n_{0}{ }^{1}$ (en pointillée) avec la courbe $n_{0}{ }^{1}$ (voir figure 8 ). La deuxième solution consiste dans une inclinaison plus forte de la courbe $n_{0}{ }^{1}$, en diminuant le temps d'écoulement $t$; c'est-à-dire par une diminution du volume d'eau, par diminution de la section de la chambre d'eau ou du diamètre de la conduite, Ce dernier moyen est limité par une certaine valeur admise de perte de la hauteur de pression. Mais un moment d'inertie plus fort augmente le prix de la machine.

La solution graphique exige peu de temps et a l'avantage d'ètre comprise très facilement. Jasse donne une méthode analytique suffisamment exacte pour la pratique dans l'article "Le temps d'emballement d'une turbine hydraulique "paru dans $E$. et $M .1922$, no 50 , page 585 . La formule $(9 b)$ employée dans la formule 4 :

$$
\frac{\omega}{\omega_{0}}=1+\left(1-\frac{\mathrm{M}}{\mathrm{D}_{0}}\right) \cdot\left(1-e^{-\frac{t}{\mathrm{~T}}}\right)
$$

Nous avons :

$$
\omega=\frac{\pi \cdot n_{\mathrm{ht}}}{30}=\frac{\pi \cdot n_{\mathrm{h}}}{0,945 \cdot 30}=\frac{\pi \cdot n_{\mathrm{h}}}{28,4}
$$

$\omega_{0}=$ vilesse normale.

$l=t_{\mathrm{h}}$ de la fig. 8 et de la formule (7).

La "constance mécanique du temps" de Jasse est: $\mathrm{T}=\mathrm{I} \cdot \frac{\omega_{0}{ }^{2}}{\mathrm{D}_{0}}$. Cette formule permet de calculer ensuite le $\mathrm{GD}^{2}\left(\mathrm{PD}^{2}\right)$ nécessaire pour ralentir l'augmentation de vitesse de $n_{\mathfrak{h}}$ (tours pendant $t_{\mathrm{h}}$ secondes.
Quand on se sert de la méthode graphique, on dessine d'abord la courbe de vitesse $n_{0}$, correspondant au moment d'incrlie admis pour les condilions de régulation. Ensuite on dessine la courbe de vitesse maximum, $n_{0}{ }^{1}$. Le point de coupure $1 / \mathrm{ne}$ nous donne d'abord pas salisfaction, $n_{h}$ est encore trop élevé. Il faut lenir compte, en supposant que $n_{\mathrm{h} 1}$ soil la vilesse maximum, que la courbe $n_{0}{ }^{1}$ nous est domée par les dimensions principales de la centrale el qu'elle est constanle. Le point $\mathrm{H}_{1}$ recherché de la vitesse maximum $n_{\mathrm{h} 1}$ doit done se trouver sur la courbe $n_{0}{ }^{1}$. Son abscisse est par conséquent donnée el la courbe de vitesse du groupe doit passer par le point $\mathrm{H}_{1}$. Par le rapport $M=I \cdot \frac{d_{\omega}}{d_{\mathrm{t}}}$ est déterminé la proportion entre le temps el le moment d'inertie. Soit $\mathrm{GD}^{2}$ lè moment d'inerlie primitif choisi, avec lequel nous établissons la courbe de vitesse $n_{0}$; GD ${ }_{1}^{2}$ le moment d'inertie plus élevé recherché, permellant une augmentation de vitesse à la valeur $n_{\mathrm{h} 1}$ dans le temps $t_{\mathrm{h} 1}$ seulemenl est donné par la formule :

$$
\mathrm{GD}_{1}{ }^{2}=\mathrm{GD}^{2} \cdot \frac{t_{\mathrm{h} 1}}{t_{\mathrm{h}}}
$$

Il résulte du texte ci-dessus, que pour des installations à haute pression avec des conduites plus longues une fermeture rapide immédiatement devant la turbine par un clapet ou par une vanne, actionnée hydrauliquement, est impossible à cause d'un sureffort dangereux des conduites (coups Bélier). De même il ne faut pas penser à une fermeture rapide des vannes dans le château d'eau (réservoir de mise en charge) ou réservoir d'eau à la prise I d'eau); car le temps nécessaire à l'écoulement de l'eau dans la conduite serait beaucoup trop long par rapport à l'augmentation rapide de la vitesse jurqu'à la vitesse d'emballement. I)ans la figure 8 par exemple, la courbe $n_{0}{ }^{1}$ serait à cause de la lente diminution de la hauteur de pression si peu inclinée, que son

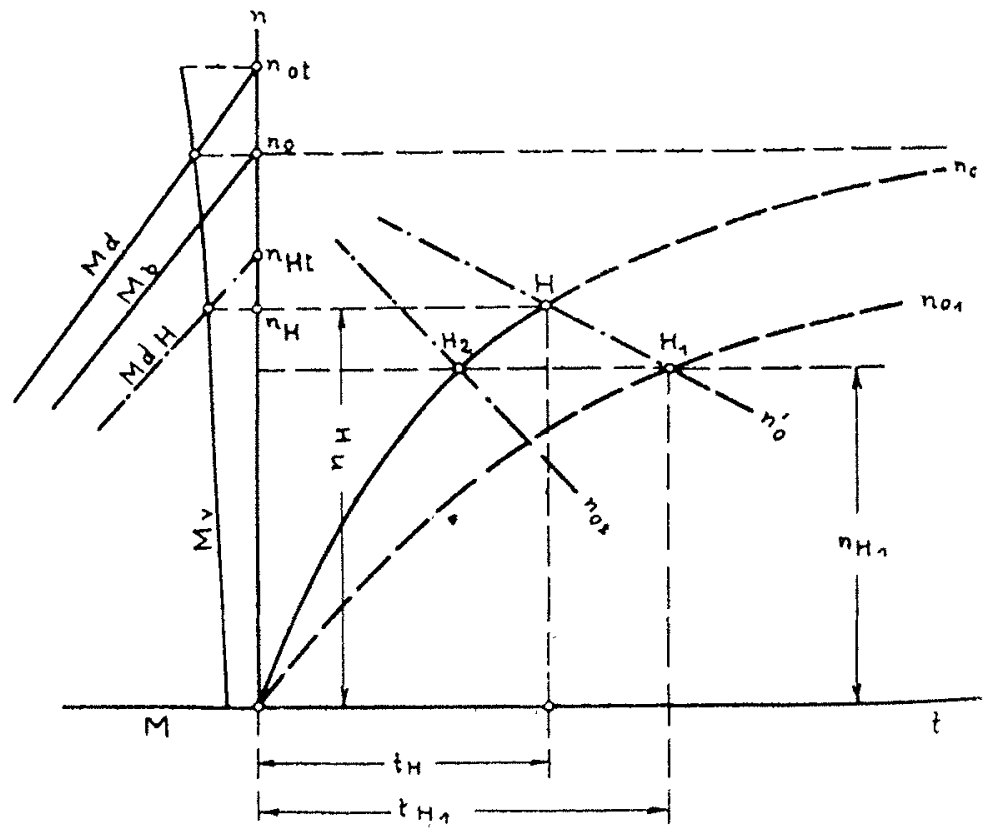

lig. 8.- Variation du temps d'emballement

point de croisement $H$ avec la courbe $n_{0}$ pratiquement ne correspondrait plus à aucune diminution de la vilesse. Les conditions pour des installations à haute pression seronl examinées cidessous pour la centrale "Walchensee" (Bavière) (voir E. T. L, 1922, numéro spécial édité à l'occasion de l'Assemblée annuelle du V. D. E. à Munich, page 15 ; Electro-Journal 1922, no. 5, page 126. En outre $\%$. V.I . I. 1921, no 27, page 707 , les lurbines Pelton sur le "Glomfjord »). 
Les turbines Pelton, entrainant des alternateurs de traction (chemins de fer) donnent une puissance de $18.000 \mathrm{CV}$ pour une vilesse de $n=250$. Le $\mathrm{GD}^{2}\left(\mathrm{PD}^{2}\right)$ est de $600 \mathrm{tm}^{2}$, et le moment d'inertie $\mathrm{J}=153.000 \mathrm{kgms}^{2}$. La courbe de vitesse (fig. 9) est trouvée par la méthode connue. Elle démontre que l'augmentalion de vitesse de $20 \%$ est déjà atteinte en 1,8 sec. et la vitesse d'emballement ( $67 \%$ augmentation) en 20 secondes. La conduite d'eau d'une longueur de $450 \mathrm{~m}$. ( $\mathrm{H}=192 \mathrm{~m}$.) a un diamètre de $2.250 \mathrm{~m} / \mathrm{m}$. à la partie supérieure (château d'eau ou réservoir de mise en charge) ; à la partie inférieure il est de $1.850 \mathrm{~m} / \mathrm{m}$. L'écoulement de l'eau de la conduite, d'un volume total de $1.430 \mathrm{~m}^{3} \mathrm{par}$ une section totale de la tuyère de $0,135 \mathrm{~m}^{2}$ nécèssite 333 secondes. Il est à signaler que la conduite alimente deux turbines, mais il faut compter avec le cas défavorable d'une seule turbine en service. Si on fermait l'entrée d'eau de la conduite au moyen des clapets rapides, en cas d'emballement, la vitesse serait en 10 secondes $n_{0}{ }^{1}=405$ pour une hauteur de pression de $181 \mathrm{~m}$. Une limitation de la vilesse par ce moyen n'est donc pas possible, car la diminution de la pression par rapport au temps d'emballement ne joue aucun rôle.

Si par contre le déflecteur du jet d'eau fonctionne automatiquement après une augmentation de la vitesse de $20 \%$, de telle sorte que le jet est complètement détourné en deux secondes, c'est-à-dire si le moment d'accélération $M$ dans l'intervalle diminue à zéro, la courbe de vilesse $\mathrm{CD}$ est trouvée graphiquement par la méthode connue (de $0,5 \mathrm{sec}$.̀̀ $0,5 \mathrm{sec}$.). La vitesse maximum devient 322 tours, c'est-à-dire $29 \%$ au-dessus de la vitesse normale ou $8 \%$ au-dessus de la vitesse atteinte au moment où intervient automatiquement le dérivateur de jet d'eau (déflecteur). La fatigue des parties tournantes est ramenée de 2,77 $\left(n_{0}=416\right)$ à 1,67. Il n'y a aucune difficulté de disposer les dérivateurs (déflecteurs) de telle façon, qu'en cas d'emballement ou d'autres accidents (dérangements) de service, les déflecteurs seront découplés du mécanisme du régulateur par pression d'huile. Ils agissent indépendamment de celui-ci sous l'influence de poids, ou actionnés rapidement par la pression d'eau de la conduite. Il est absolument nécessaire que ce dispositif de sécurité soit complètement indépendant du régulateur à pression d'huile, etc... Contre-tuyères, des tuyères de frein dont l'admission est inefficace pour arrêter l'emballement des turbines (le contre couple est trop petit), servent seulement pour arrêter le groupe après la

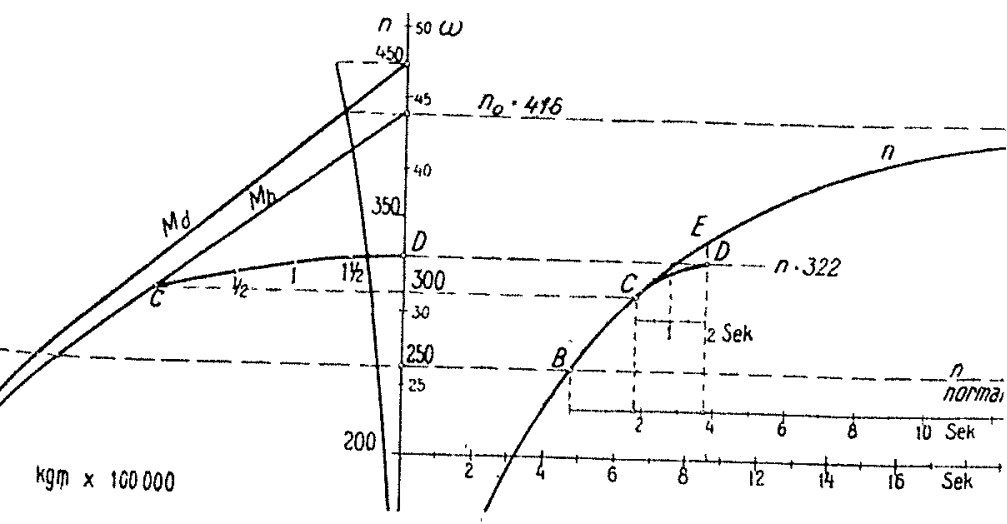

Fig. 9. - Caractéristiques de lurbines à haute pression (Centrale de Walchensee)

fermeture des vannes d'arrivée. Et l'inslallation de turbines de frein avec leurs régulateurs tournant dans le sens inverse est même pour des très grosses unilés trop cher (voir la centrale de Slura Di Viu de S. A. Alta (Italie) (lurbines à jet libre à deux luyères tournant en sens inverse des turbines spirales de $4.000 \mathrm{CV}$ ). Un développement dans ce sens pourrait permeltre des économies importantes des alternateurs el éviterait un dépassement de la vitesse admise, sans mesures spéciales.
On trouve une courbe de vitesse semblable pour les turbines Francis de 24.000 CV de la centrale de Walchensee, pour une vitesse $n=500$ et le $\mathrm{GD}^{2}=160 \mathrm{t} / \mathrm{m}^{2}$. L'écoulement de l'eau dans la conduite se fait en $261 \mathrm{sec}$. Dix secondes après la fermeture rapide des vannes la chute est toujours encore de $178 \mathrm{~m}$. correspondant à une vitesse maximum de 800 en chiffre rond. Il n'en résulte donc aucune sécurité par la fermeture rapide des vannes.

Un frein mécanique permettant d'arrêter le groupe ne peut non plus être pris en considération.

Pour des turbines Francis à haute pression, seul un clapet devant la turbine (fermant en deux à trois secondes) en liaison avec

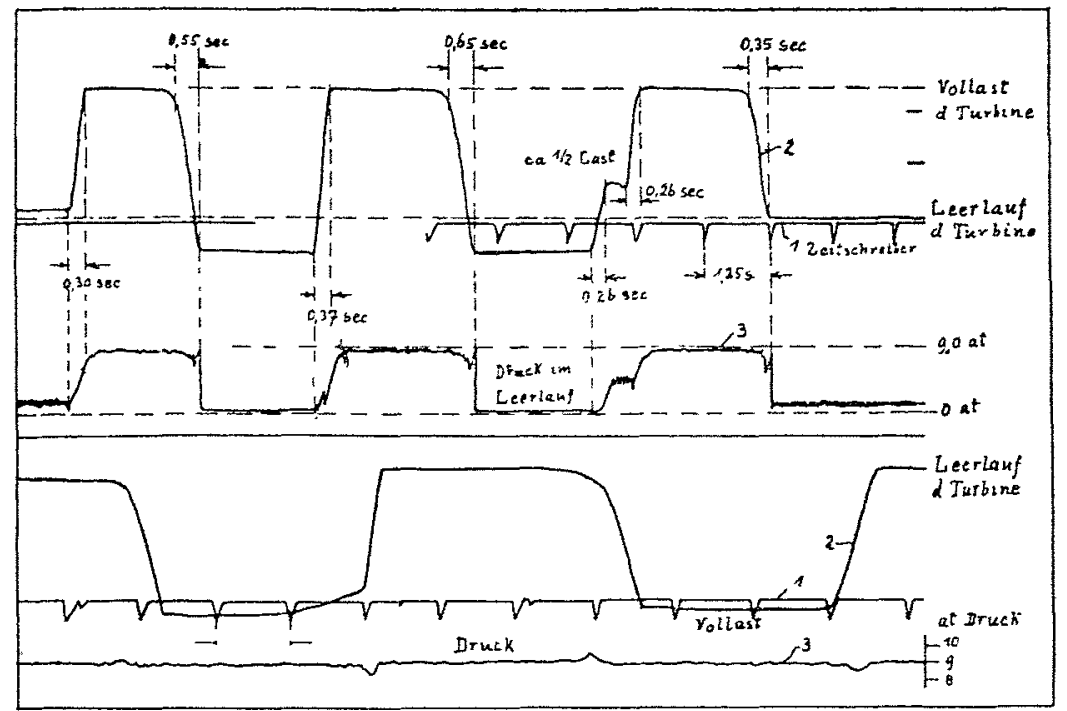

Fig. 10. - Variation de la pression en cas de régulation par chemin détourné "courbe supérieure : devant " la turbine, courbe intérieure: dans la conduite devant la vanne.

un dispositif d'écoulement par le còté (fonctionnant en mème temps que le clapet et indépendamment du régulateur) sont d'une utilité pratique. Il est vrai, qu'on a malgré tout de nouveau une possibilité de dérangement en liant ces deux dispositifs, qui dans certaines circonstances peuvent provoquer une rupture de la conduite. Dans beaucoup de cas, surtout applicables à des installations déjà existantes, on devrait devant la turbine prévoir une évacuation automatique d'eau par le côté au moyen d'un clapet hydraulique au lieu d'une fermeture rapide des vannes. En cas de danger, ce clapet sera ouvert automatiquement ou actionné du poste de commandement.

La nouvelle vanne de distribution (clapet multiple) de Poebing paraît dans ce domaine avoir trouvé son avenir comme véritable organe de régulateur. Ce régulateur est construit de manière que le débit d'eau d'entrée de la turbine est réduit de la quanlité d'eau sortant par la conduite latérale. La somme des deux parties (c'est-à-dire lè débit total dans la conduite d'arrivée) l'eau actionnant les aubes, plus l'eau sortant par la conduite latérale, est toujours constante. La distribution d'eau sur la turbine et la sortie d'eau varient suivant la disposition de la vanne.

Le pression dans la turbine change naturellement par rapport à la pression totale (action totale sur les anbes) d'un maximum à un minimum (ouverture complète de la conduite latérale). Ce dispositif est connu sous le nom de régulation "Esibe ", voir Wasserkraft, $1921, \mathrm{n}^{\circ} 17$, page $220 ; 1922, \mathrm{n}^{\circ} 21$, page $10 ; \mathrm{n}^{\circ} 5$ page 92; E. $M ., 1922$, n' 1, page 9, conférence par M. Budau, Professeur. Vienne, le 21 novembre 1922, à la Société des Ingénieurs. On propose d'appeler ce dispositif " régulation par che- 
min détourné ". Si cette nouvelle vanne multiple, actionnée par un contact centrifuge par bouton de commande ou autres déclenchements instantanés et ramenée à la position de marche à vide par leffet de poids indépendants ou par pression d'eau, laugmentation de la vitesse ne sera pas sensible. Nous avons alors à peu près les mèmes conditions que dans les turbines à filet libre avec dispositif de dérivation du filet (turbine à libre jet). Cette nouvelle vanne peut ètre ramenée d'une position extrême à l'autre de $1 / 2$ à $1 / 10$ secondes, sans qu'il n'y ait aucun danger pour la conduite. Une vanne "Poebing " construile comme vanne de régulation rapide et soumise à des essais a donné les diagrammes de pression suivant fig. $n^{\circ} 10$. L'enregistrement a eu lieu avec un nouvel appareil de haule sensibilité de "Forderreuther ". Il est muni de trois aiguilles à encreur. La ligne $n^{\circ} 1$ indique le temps, la ligne $n^{\circ} 2$ la position de la vamne et la ligne $n^{\circ} 3 \mathrm{la}$ pression. Toule erreur possible provenanl des frottements; l'effet de masses, est paré par des dispositifs spéciaux. La série de courbes supérieures de la figure 10 montre l'indication de pressions en avant de la turbine, c'est-à-dire derrière la dérivalion de la conduite d'écoulement latérale pour des temps d'ouverture et de fermeture de pleine charge à marche à vide el sens inverse entre 0,65 et 0,35 secondes. La pression devant la turbine varie immédiatement avec la position de la vanne. Pendant la marche à pleine charge de la turbine, cette vanne ferme complètement la sortie d'eau latérale; pour la marche à vide-par contre, elle la libère de manière que pour un débit constant, la pression d'eau devant la turbine (qui est toujours réglée sur l'ouverture pleine), soit suffisante pour maintenir la vitesse normale.

Par contre, le diagramme inférieur montre la pression dans la. conduite d'eau devant la vanne à distribution multiple (vanne Poebing). L'augmentation de la pression en cas de fermeture rapide de la turbine, c'est-à-dire en ouvrant la conduite d'écoulement latérale, est de moins de $10 \%$; elle n'est donc pas dangereuse pour la conduite. Ces essais ont été obtenus sur un modèle de grande centrale, rédúte à petite échelle. La longueur de la conduite était de $10 \mathrm{~m}$. avec un diamètre de $65 \mathrm{~m} / \mathrm{m}$., il en résulte une vitesse de l'eau de $5,4 \mathrm{~m}$. sec. Il est à remarquer que pour le diagramme supérieur (devant la turbine) la variation de pression est régulière; en cas d'ouverture rapide de la turbine, nous constatons une augmentation de pression momentanée, et qui provoque une accélération de la turbine; en cas de décharge nous avons une courte diminution de pression. On pourrait facilement arriver à une pression constante dans la conduite d'eau, ou la faire varier avec la position de la vanne.

Une sortie d'eau de côte provoquée par la mise en action d'une soupapé, sans chemin détourné, comme c'est le cas pour là régulation de "Poebing " rendra sans valeur les réserves faites par Beck (voir E. K. B., 1922, no 19, page 226, et E. T. Z., 1923, no 12 , page 274).

Beck dit avec raison que les stations à haute pression avec des conduités longues doivent être calculées à une vitesse d'emballement bien supérieure à 1,8 fois la vitesse normale. La variation de pression dans la conduite peut osciller pendant un temps de beaucoup supérieur au temps nécessaire à l'emballement. Il est donc possible que la turbine accélère sous une pression supérieure à la pression de service normal (pression de marche normale. (Si par exemple dans la conduite se produisait une augmentation de pression de $50 \%$ et si la turbine ne pouvant pas se fermer complètement, la vitesse d'emballement devrait être de 2,2 au lieu de 1,8 fois la vitesse normale. Cela veut dire que la fatigue du matériel serait $50 \%$ au dessus de la sécurité de 1,8 habituelle à cause de la force centrifuge.

Des moyens pour la fermeture des appareils de commande par des dispositifs automatiques indépendants, accouplés au régulateur à main, ne possèdent pas une sécurité pleine et entière contre l'emballement. Car l'emballement n'est pas nécessairement provoqué par un défaut dans le régulateur; il peut résulter de la casse ou d'un coincement dans les engrenages de la turbine, la conséquence immédiate est l'impossibilité de fermer les aubes ou les tuyères. Inversement, le régulateur défectueux peut empêcher la fermeture par d'autres moyens. Des régulateurs de sécurité sont par contre tout à fait indiqués là où il s'agit seulement d'une sécurité contre un emballement sans régulation automatique de la vitesse, par exemple pour des installations asynchrones.

Une solution générale de la question sous forme d'une formule empirique par exemple : "à partir de quelle longueur de conduite une fermeture rapide peut-elle empêcher l'emballement de la turbine "ne peut être donnée; car la courbe, donnant l'augmentation de la vilesse monte suivant le moment d'inertie résultant des conditions de régulation. Des intervalles d'une demi-seconde jouenl déjà une influence considérable. L'importance du problème, d'abandonner la condition actuelle d'avoir un coefficient de sécurité contre la fatique des parties tournanles, surtoul pour des grandes unités à grande vitesse, mérite une étude approfondie dans chaque cas. Les moyens pour arriver à ce but el décrits ci-dessus sont résumés ci-après.

Pour des basses et moyennes chutes et puits de turbine onvert ou arrivée d'eau en conduite : une fermeture rapide des vannes d'arrivée, si nécessaire en liaison avec un disposilif d'aération du tuyau d'aspiration, actionné par contact centrifuge (évelltuellement avec dépendance du disjoncteur de l'alternateur, d'un relais de tension à maxima; thermomètre à contact) avec possibilité à commande à distance du poste de commandement. Dans ce but, le poste sera muni de tachymètres à distance pour chaque groupe; de même de thermomètres à distance pour les alternateurs el les gros transformateurs. Malheureusement, on ne tient pas toujours compte dans les nouvelles centrales de ces appareils de contrôle.

Pour les petites centrales, un dispositif d'aération du tuyau d'aspiration éventuellement seul peut suffire et pour des centrales avec des alternateurs asynchrones, c'est le seul dispositif pour régler les turbines.

Pour les turbines à filet libre, la protection contre lemballement consiste dans l'installation d'un dispositif automatique indépendant pour dériver le filet. La fermeture rapide des vannes sert ici uniquement comme protection contre une rupture de la conduite.

Les turbines Francis à haute pression seront avantageusement protégées par les vannes spéciales "Poebing " actionnées indépendamment et avec conduite latérale d'écoulement largement dimensionnée pour ouverture rapide.

S'il existe des freins spéciaux pour arrêler les groupes, lous ces dispositifs de sécurité sonl à relier avec eux.

En outre, on peut prévoir la disposition des groupes de telle sorte, qu'en cas d'éclatement d'un groupe, les aulres souffrent aussi peu que possible; c'est-à-dire à les disposer dans un seul axe, avec bâtiment d'appareillage à une extrémilé du bâtiment. Il faut également avoir soin d'éviler des poutres, des ferrures, etc... au-déessus de l'alternateur pour éviter des démolitions de parties importantes du bâtiment. Chaque centrale a naturellement ses particularités; il faut juger chaque cas, suivant les conditions du terrain, etc...

Traduction $E$. et $M_{.}$, n$^{\circ} 39$, page 566 et no 40 , page 582 . 